\title{
Single-cell characterization of neovascularization using hiPSC-derived endothelial cells in a 3D microenvironment
}

\author{
Simon Rosowski ${ }^{1}$, Caroline Brähler ${ }^{1}$, Maren Marder ${ }^{1}$, Misao Akishiba ${ }^{1}$, Alina Platen ${ }^{1}$, Siegfried \\ Ussar $^{2}$, Fabian Theis ${ }^{3,4}$, Sandra Wiedenmann ${ }^{\$ 1}$, Matthias Meier ${ }^{\$ 1,5}$
}

${ }^{1}$ Helmholtz Pioneer Campus, Helmholtz Zentrum München, Neuherberg, Germany

${ }^{2}$ Institute for Diabetes and Obesity, Helmholtz Diabetes Center, Helmholtz Zentrum München, Neuherberg, Germany

${ }^{3}$ Institute of Computational Biology, Helmholtz Zentrum München, Neuherberg, Germany

${ }^{4}$ Department of Mathematics, Technical University of Munich, 85748 Garching bei München, Germany

${ }^{5}$ Medical Faculty, Technical University of Munich, Munich, Germany

\$ Corresponding authors:

Dr. Matthias Meier (lead contact)

Sandra Wiedenmann

Email: matthias.meier@helmholtz-muenchen.de

Email: sandra.wiedenmann@helmholtz-muenchen.de 


\begin{abstract}
The formation of vascular structures is fundamental for in vitro tissue engineering. Vascularization can enable the nutrient supply within larger structures and increase transplantation efficiency, which are currently limiting factors in organoid research. We differentiated human induced pluripotent stem cells toward endothelial cells in 3D suspension culture. To investigate in vitro neovascularization and various 3D microenvironmental approaches, we designed a comprehensive single-cell transcriptomic study. Time-resolved single-cell transcriptomics of the endothelial and co-evolving mural cells gave insights into cell type development, stability, and plasticity. Transfer to a 3D hydrogel microenvironment induced neovascularization and facilitated tracing of sprouting, coalescing, and tubulogenic endothelial cells states. During maturation, we monitored two pericyte subtypes evolving of mural cells. Profiling cell-cell interactions between pericytes and endothelial cells confirmed in vivo angiogenic signaling and emphasized new cytokine signals during tubulogenesis. Our data, analyses, and results provide an in vitro roadmap to guide vascularization in future tissue engineering.
\end{abstract}

\title{
Keywords
}

single-cell mRNA sequencing, stem cell-derived endothelial cells, culturing technologies, neovascularization

\section{Introduction}

Endothelial cells (ECs) form the inner luminal epithelium of vascular structures, including arteries, veins, and lymphatic vessels ${ }^{1}$. Apart from core nutrient and oxygen transport functions, the vascular system enables immune cell trafficking, vasomotor tone, and wound healing ${ }^{2}$. To fulfill the different homeostatic functions, ECs exhibit a high degree of plasticity to form differently sized fenestrae and branched vessel structures, recruit organ-specific scaffold cells, or remodel the extracellular matrix composition of the vascular bed ${ }^{3,4}$. The developmental factors determining EC formation, maturation, and specification are not fully revealed yet.

In vitro cell culture systems with patient and stem cell-derived ECs have become indispensable for vascular research to overcome the paucity of longitudinal studies in patients, reducing the biological complexity. In particular, stem cell-derived ECs are of interest because they provide access to the early development stage of vascular structures involving cell type formation and specification $^{5}$. In vivo, ECs evolve from mesoderm-derived progenitors (angioblasts) in response to FGF2 and VEGFA signals from the adjacent visceral endoderm ${ }^{6}$. Stem cell differentiation 
protocols recapitulate in vivo development by first inducing mesodermal progenitor cells with BMP4 and GSK3 $\beta$ inhibitors ${ }^{7}$, and subsequently ECs by adding VEGFA ${ }^{8}$. Several variations of the differentiation protocols have been reported ${ }^{9}$, where the differences between the resulting ECs remain unresolved. Stem cell-derived ECs do not show a direct correlation with any organspecific ECs and it remains unclear which developmental stage they reflect ${ }^{10}$. To closely resemble the in vivo microenvironment in a dish, 3D stem cell culture formats were introduced, which enable stem cell-derived ECs to form tubes ${ }^{11}$. Moreover, stem cell-derived ECs can fully self-assemble into blood vessels in an organoid-like format, including mural cells, i.e., smooth muscle and pericytes lining the outer surface of the vessel endothelium ${ }^{12}$. In all culture formats, mural cells co-evolve during EC differentiation ${ }^{13}$, which implies inherent strong cell-cell communication during differentiation and maturation.

During embryonic development, immature ECs coalesce and undergo tube formation to produce a vascular plexus ${ }^{14}$, which further differentiates into the specific vessel types. Single-cell transcriptomic analysis of the in vivo neovascularization process was performed by extracting cells from laser-induced choroid lesions in mice ${ }^{15}$. The data depicted a highly heterogeneous process with multiple transcriptomic EC stages and types, including phalanx and tip cells, with associated distinctive metabolic profiles. Comparable single-cell transcriptomic analyses in a less complex in vitro microenvironment with stem cell-derived ECs is not available but would add fundamental knowledge on the formation of vascular structures.

In this study, we used single-cell transcriptomics to sequentially investigate the development and neovascularization of human induced pluripotent stem cells (hiPSCs) in an in vitro 3D microenvironment. In the first step, we used single-cell transcriptomics to explore the differentiation trajectory of co-evolving ECs and mural cells in a 3D suspension cell culture format. Comparison of single-cell transcriptomics of ECs, evolved of a monolayer and 3D suspension culture, revealed differences in ECM gene expression and optimal differentiation parameters. In the second step, the single-cell transcriptomics approach was used to analyze the neovascularization in the heterogeneous $3 \mathrm{D}$ suspension culture upon transfer into a hydrogel culture. Ligand-target links between ECs and subcellular pericyte populations were predicted from the single cell transcriptomes during vessel maturation. Our data and analysis provided the resources for future EC specification studies and tissue engineering approaches. 


\section{Results}

Single-cell analysis of endothelial differentiation in a 3D suspension culture

To investigate the differentiation of hiPSCs into ECs in a 3D cell culture format at the single-cell level, we adopted the chemical two-step induction protocol ${ }^{11,12,16}$. Therefore, hiPSCs were differentiated towards the mesoderm germ layer and EC development was induced in the second step (Fig 1a). In suspension, the 3D cell cultures were stable over the differentiation and grew from a diameter of $150 \mu \mathrm{m}$ to $300 \mu \mathrm{m}$. While hiPSC-derived aggregates exhibited a uniform spheroidal shape, aggregates from day four showed a more prolate shape. On day nine, $33.2 \%$ of the cells expressed the endothelial marker CD31 (PECAM1), with a standard variation of $5.3 \%$ over three biological repeats (Fig. S1a). To reconstruct EC development in the 3D suspension culture and define time-resolved cell composition, we performed single-cell mRNA sequencing (scRNA-seq) analysis on 22,192 cells (see Table S1 for details). Upon Leiden clustering ${ }^{17}$, six cell clusters were identified. With the progression of the differentiation process, the recorded single-cell transcriptomes changed, as indicated by the time-dependent emergence of distinct cell clusters (Fig. 1b). All cell clusters could be assigned to cell types by matching known mesodermal and endothelial developmental markers to the differentially expressed genes (DEGs) in the respective cluster (Fig. 1c). The cell populations were assigned to pluripotent stem cells (cluster 1 ), mesenchymo-angioblasts (cluster 2), mural cells (cluster 3 ), cells undergoing mesenchymal-endothelial transition (MEndoT) (cluster 4), angioblasts (cluster 5 ), and epithelial cells (cluster 6). At the start of differentiation (day 0), the cell population consisted of homogenous undifferentiated hiPSCs, where over $96 \%$ of the cells expressed the pluripotency markers OCT4, SOX2, and NANOG. Cells assigned as mesenchymo-angioblasts appeared on day three of differentiation and expressed markers for the lateral plate mesoderm $^{18}$, including HAND1, MESP1, and APLNR. Mural cells observed on day six of differentiation showed reduced HAND1 expression level, while smooth muscle marker ACTA2, pericyte marker $P D G F R B$, and mesenchymal marker COL1A1 were consistently expressed. MEndoT cells in cluster 4 expressed the same markers but also included the endothelial markers PECAM1, CDH5, and ESM1. Only a small fraction (0.9\%) of epithelial cells was observed (Fig. 1d). Immunohistochemical staining of 3D suspension cell cultures with the markers PECAM1 and PDFGRB showed de-mixing of the two cell populations; however, there was no induction of vessel formation, supporting the angioblast cell state (Fig. S1e). To test the robustness of the differentiation approach at the single-cell level, we sequenced the cells from day nine of two independent differentiation experiments. In both cases, a bimodal distribution of 
angioblasts and mural cells was observed, with comparable distribution numbers (Fig. S1b and

c).

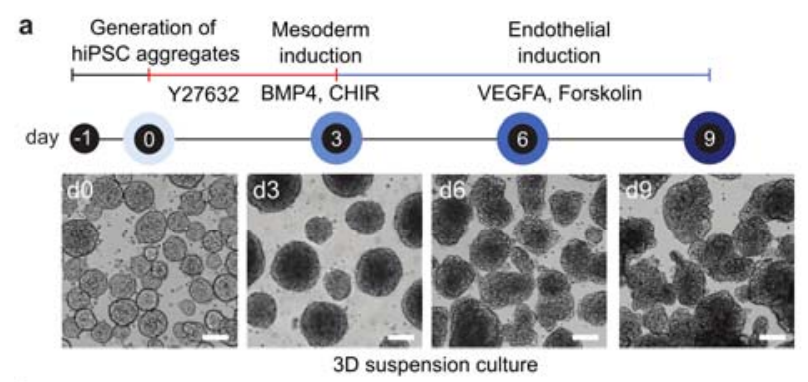

b
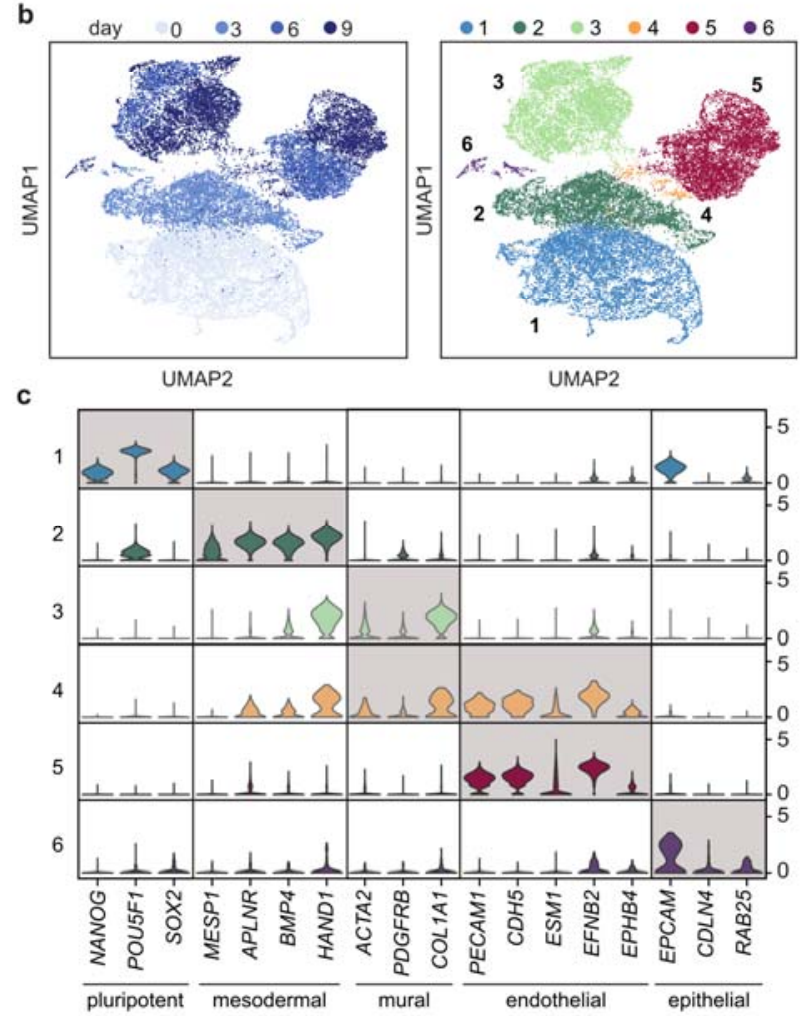

d

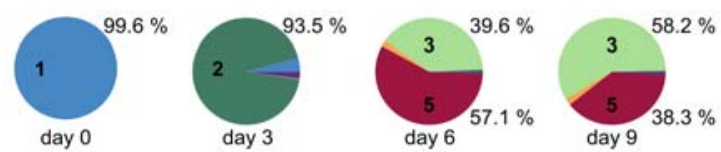

Fig. 1 | Single-cell transcriptomics reveals the differentiation trajectory of hiPSCs into endothelial cells in 3D suspension culture. a, Schematic of the endothelial differentiation timeline with sampling points and chemical induction protocol. Bright-field images show representative 3D suspension cultures at the corresponding time point. Scale: $100 \mu \mathrm{m}$. b, UMAP plot of the single-cell transcriptomes. Left: Light to dark blue denotes the time points of sampling. Right: Six unique cell clusters were identified during the endothelial differentiation, 1 : hiPSCs, 2: mesenchymo-angioblasts, 3: mural cells, 4: MEndoT, 5: angioblasts, 6: epithelial cells. c, Violin plot shows the cluster expression levels of differentially expressed genes for the six cell clusters and the commonly used cell markers for cell type assignment. d, Cell type distribution analysis along the differentiation trajectory. 


\section{Time trajectory of endothelial development}

To resolve the time-dependent relationships of the cell clusters, we performed a dynamic RNA velocity analysis ${ }^{19}$ and identified the dynamic driver genes (DDG) in endothelial differentiation ${ }^{20}$. For this, we first calculated the latent time based on the balance of spliced and unspliced RNA transcripts within the single-cell transcriptomes (Fig. 2a). Indeed, the theoretical latent time matched true chronological differentiation times (compared to Fig. 1a). The corresponding RNA velocity streamlines indicate two differentiation routes. The differentiation route for angioblasts and mural cells evolved from mesenchymo-angioblasts. Also, velocity streamlines between mural and angioblasts via the intermediate cells undergoing MEndoT indicated a relevant degree of plasticity of the cell types at this stage of development. Evaluation of cell cycle states showed that angioblasts were entirely in the G1 phase, whereas approximately $50 \%$ of the mural cells were in $\mathrm{G} 2$ and $S$ and thus proliferating (Fig. S1d). Subsequently, we plotted the DDGs along the latent time to trace the central genes for the development of angioblasts in 3D (Fig. 2b). Similar to the DEG analysis, cluster-specific DDGs were identified. The list of DDGs for each cell cluster is given in SI File. The top DDGs for the mural cell progenitors were involved in cell migration, attraction, or repulsion (UNC5C, SLIT3, and TGFB2) ${ }^{21,22}$. For ECs, known developmental genes of vasculogenesis were upregulated, including the VEGF receptors ( KDR, FLT1) and the interacting receptors TIE1 and $T E K^{23,24}$. To infer the transcription factors (TFs) controlling the development of angioblasts and mural cell progenitors, a transcription factor enrichment analysis (TFEA) was performed on the DDGs (Fig. 2c). The highest-ranked TFs for EC development were $B C L 6 B^{25}, E T S 1^{26}, E L K 3^{27}, E R G^{28}$, and members of the SOX family. All of them are reportedly associated with the process of early vasculogenesis with context-dependent function but integrate the VEGFA and Notch signaling pathways ${ }^{25}$. Notably, the extracted TFs are only responsible for the development of the two identified cell types, but not for neovascularization since images of sections of 3D cell cultures taken on days six and nine showed no vessel organization (Fig. S1e). TFEA of the DDGs for the mural cell progenitors revealed TBX18, CENPA, and HAND2 as the top regulatory $\mathrm{TFs}^{29}$. In particular, TBX18 has been recently identified in mice to be selectively expressed by all pericytes and vascular smooth muscle cells within the retina, brain, heart, skeletal muscle, and adipose fat depots ${ }^{30}$. It has to be considered, that the velocity analysis with spare time points has to be understood as transcriptional correlation rather than real dynamics. Nevertheless, turn-on and -off of genes derived from the velocity analysis match with the expectation, for example for the cell cycle regulator HMGA2. In mural cells HMGA2 is in the turned-on state, whereas in angioblasts in the turned-off state but still detectable. 

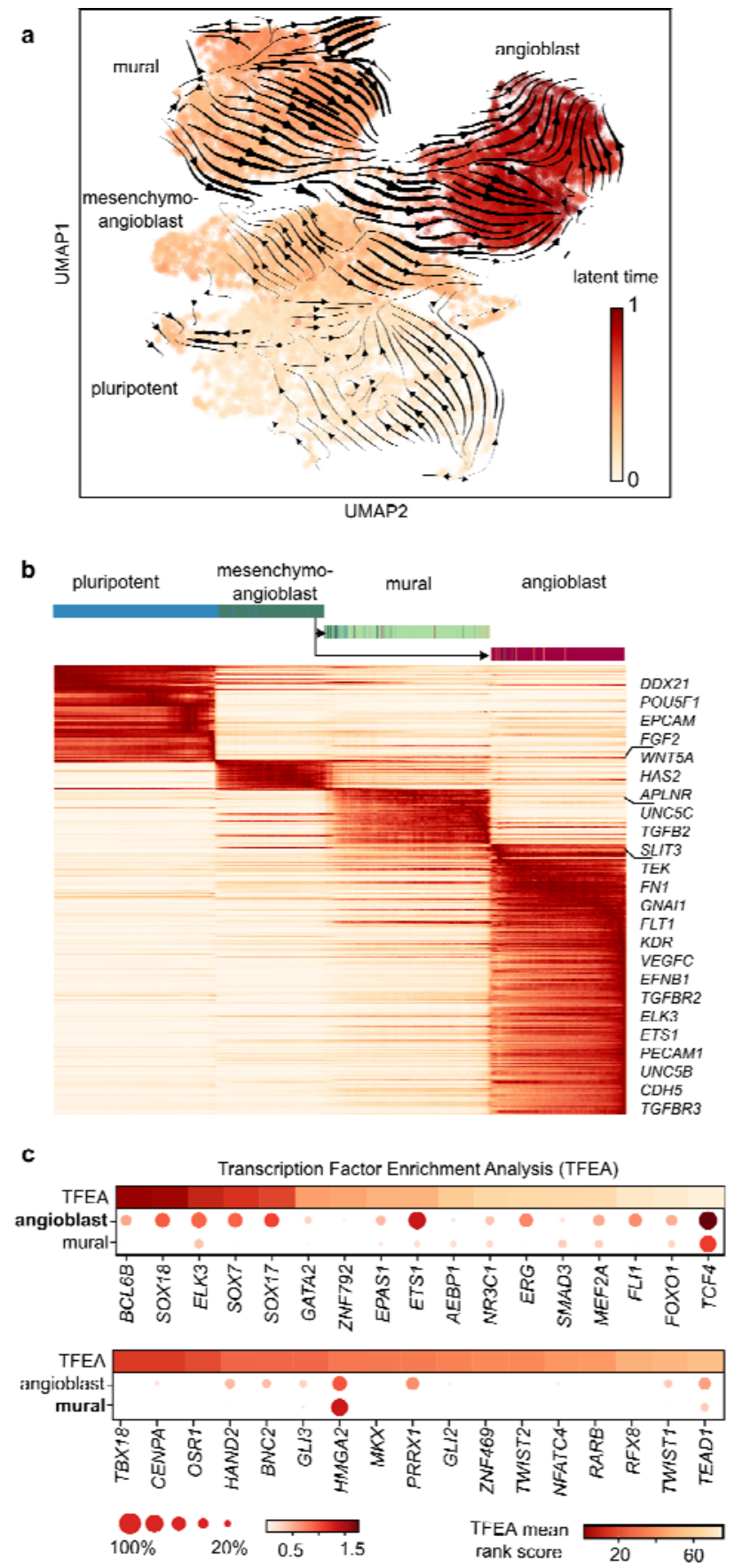

Fig. 2 | Transcriptomic dynamics predict the differentiation path for endothelial and mural cells. a, UMAP cluster plot colored with the latent time calculated based on RNA velocity analysis. The velocity streamlines are given by the black arrows. b, The top 300 DDGs sorted according to their likelihood scores and latent time (see SI File). c, TFEA on the cluster-specific and unique DDGs for angioblasts and mural cells. The dot plots show the gene expression level in the respective single-cell cluster, whereas the upper heat-colored bar shows the TFEA score. Color intensity and dot size denote the normalized cluster mean expression and the fraction of cells expressing the gene, respectively. 


\section{Comparison of endothelial differentiation in $3 D$ versus $2 D$ cell culture formats}

In the next step, we sought to compare the in vitro development of ECs in 3D suspension culture to a previously performed stem cell differentiation approach with an adherent 2D cell culture on the single cell level ${ }^{13}$. Chemical compounds for the endothelial induction protocol were the same; however, minor concentration differences of the individual compounds existed (Table S2). Single-cell transcriptomic trajectories of the 3D and 2D differentiation approaches differed with respect to the marker expression profiles of intermediate and evolving EC and mural cell types (Fig. 3a). In the 3D suspension culture, the mesenchymo-angioblast markers (APLNR, KDR, and PDGFRA) were expressed strongly. In the 2D cell culture, the markers were either lowly expressed or even absent (Fig. S2a). To quantify the differences, we combined the two scRNAs datasets. Of the 14,383 genes, 682 and 652 showed expression level differences with a p-value lower than $10^{-100}$, in the $2 \mathrm{D}$ and $3 \mathrm{D}$ cell cultures, respectively (Fig. $\mathbf{3 b}$ ). Expectedly, the expression patterns of the ECM genes were distinct in different culture formats. Within the 2D cell culture format, ECs exhibited a strong collagen phenotype with high expression levels of basal lamina proteins, such as COL4A1/2, COL6A2, or COL18A1 (Fig. 3c). In 3D cell culture, ECs expressed hyaluronic acid and the corresponding binding proteins. Further, a GO term analysis of the DEG showed that ECs in the 2D cell culture expressed genes associated with migration and motility while Rap signaling was enriched in 3D (Fig. S2d and $\mathbf{e}$ ). In contrast, angioblasts within the 3D suspension culture upregulated cell-cell interaction and actin remodeling genes, such as CLD5, DOCK4, and CTNNB1 (Wnt signaling) ${ }^{31}$, and $R A P 1 B^{32}, R A P G E F 5^{33}$, and $R A S I P 1^{34}$ (Rap1 signaling), respectively. 
a
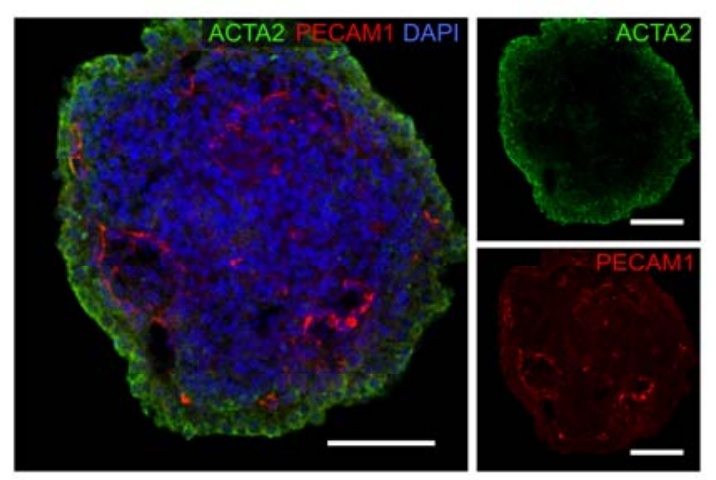

b

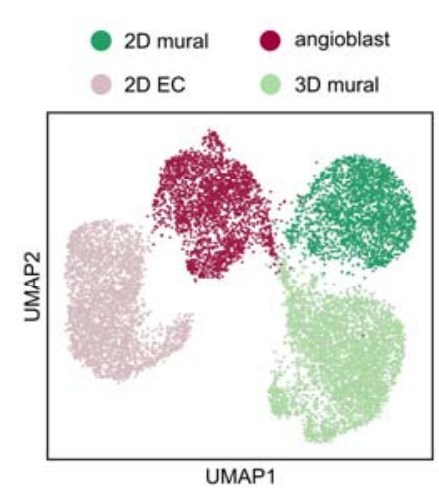

c

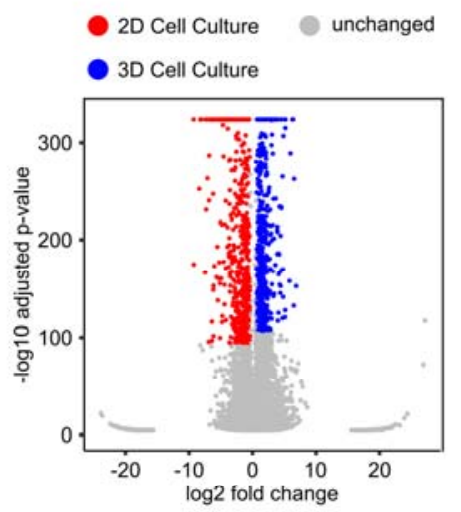

d

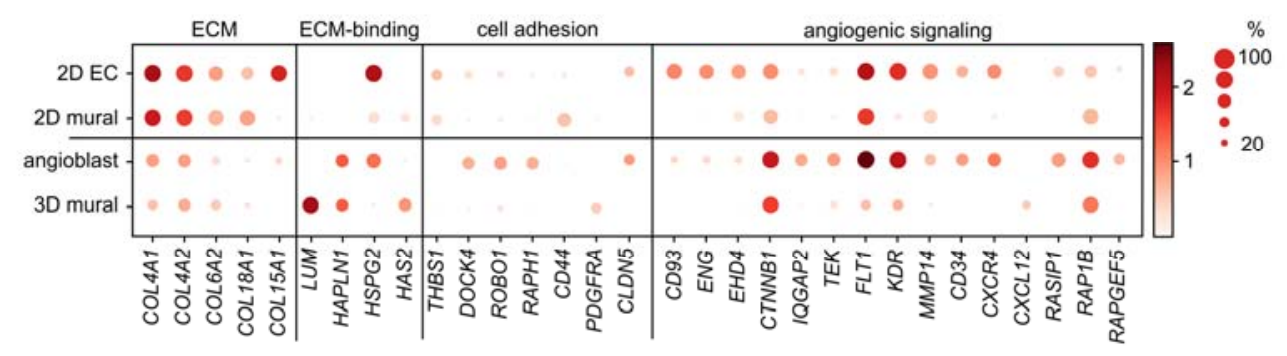

Fig. 3 | Comparison of EC differentiation in 2D and 3D cell culture formats. a, Immunofluorescence image of an aggregate from 3D suspension culture on day 9 of differentiation. Scale: $100 \mu \mathrm{m}$. b, UMAP plot of single cell transcriptomes of iPSCs derived endothelial cells differentiated on a 2D adhesion monolayer (5267 cells) and as 3D suspension culture (6492 cells). c, Volcano plot representing differentially expressed genes between ECs differentiated in 2D and $3 \mathrm{D}$ cell cultures. d, Representative different expressed genes with assigned cellular functions and biological processes. Color intensity and dot size denote for the normalized cluster mean expression and fraction of cells expression the corresponding gene, respectively.

While mural cells and ECs in the 2D cell culture format were proliferating, within the 3D cell culture, only the mural cells were cycling (Fig. S2b). This raised the question of whether the separation of the ECs from the mural cells or the transfer to a $2 \mathrm{D}$ culture format reinstates the proliferation of ECs. Indeed, FACS-sorted PECAM1 ${ }^{+}$ECs from the 3D suspension culture proliferated and could be passaged over six generations upon plating on a culture dish. Comparison between sorted PECAM1 ${ }^{+}$cells within a $3 \mathrm{D}$ and $2 \mathrm{D}$ cell culture was not possible due to the observation that ECs alone did not form a stable aggregate in suspension culture. However, the transfer of the unsorted mixed 3D aggregates to a $2 \mathrm{D}$ cell culture format on day six of differentiation led to the proliferation of ECs, demonstrating that growth arrest is associated with the $3 \mathrm{D}$ suspension culture format ${ }^{35}$. 
A further important question for cell type engineering was whether sorted ECs and mural cells maintained cell type stability after sorting. To evaluate this aspect, we investigated the ratio of $\mathrm{PECAM}^{+}$and $\mathrm{PDGFRB}^{+}$cells within sorted and unsorted 3D suspension cultures. Within sorted PECAM1 ${ }^{+} / \mathrm{PDGFRB}^{-}$cells cultures no PDGFRB ${ }^{+}$cells could be detected after six days of culturing in a monolayer culture format under EC differentiation medium (Fig. S7b and c). In contrast, PDGFRB ${ }^{+}$PECAM1 $^{+}$sorted cells lost the PDGFRB expression under the same conditions. Non-sorted cell cultures the fraction of PECAM1 ${ }^{+} /$PDGFRB $^{-}$cells increased to $72 \%$, where the fraction of PDGFRB ${ }^{+}$cells also decreased to $10 \%$ after six days of culturing in a monolayer. This finding contrasted with that in the 3D suspension culture, where the PDGFRB ${ }^{+}$ cell fraction was unchanged within the same culturing interval. In conclusion, cell-cell signals between angioblasts and mural cells lead to stabilization of the mural cell fraction.

\section{Single-cell transcriptomics of in vitro sprouting and coalescing ECs}

Stem cell-derived angioblasts generated under comparable conditions can be induced by sprouting ${ }^{12}$. We induced endothelial sprouting by transferring the 3D suspension culture into Matrigel (Fig. 4a). Sprout formation was observed within the first 12 h (Fig. S4d). Single-cell transcriptomes of the sprouting Matrigel culture were determined $48 \mathrm{~h}$ after transfer and compared to single-cell transcriptomes of cells within 3D suspension cultures kept to the same day of differentiation. ECs formed two transcriptomic subclusters within the Matrigel culture, which were separated from ECs in the 3D suspension culture (Fig. 4b). In addition, mural cells formed two transcriptomic subclusters, where one cluster overlapped with the transcriptomic state of the mural cells in the 3D suspension culture. Transcriptomes of MEndoT cells clustered with their analogs in 3D suspension culture (Fig. 4c). The expression levels of general cell type markers are shown with cluster assignments in Fig. S3a. A corresponding velocity analysis with a calculated latent time of the combined datasets indicated that the transcriptional states of ECs observed in the Matrigel culture evolved from the suspension culture, similar to the mural cells (Fig. 4d). Further, the streamlines of the velocity analysis indicated that MEndoT cells developed from the mural cells towards EC within the Matrigel microenvironment, which is consistent with the velocity analysis of the $3 \mathrm{D}$ suspension culture.

For the formation of microvessels, angioblasts within the Matrigel-embedded 3D suspension culture must migrate into the hydrogel and coalesce. To infer which genes drove the processes, we plotted the DEGs related to ECM remodeling, cell migration, cell interaction, and VEGF signaling (Fig. 4e). Based on the DEG cluster profiles and corresponding GO term analysis, EC 
clusters 5 and 6 can be assigned to coalescing (cEC) and sprouting (sEC) endothelial cell states, respectively (Fig. S2c and d). The transfer to Matrigel increased the expression levels of genes involved in migration within the ECs (e.g., AMOTL2 $2^{36}, A N X A 1^{37}$, or $Z E B 2^{38}$ ) and reduced the expression levels of genes involved in cell interactions (NECTIN2/3 or CLDN5). ECM proteins required for the build-up of a basal lamina increased in cECs $\left(C O L 4^{39}, C O L 18^{40}\right)$. The expression levels of integrin changed only slightly between the three EC transcriptional states. Moreover, only the collagen type 1 metalloproteinase (MMP) 2 was gradually upregulated upon cell state transversion from angioblasts into cECs, whereas MMP9 and MMP14, which are known to promote motility and tubulogenesis, respectively, were downregulated ${ }^{41,42}$. The most obvious was the downregulation of genes related to the VEGF signaling network within cECs and sECs, except for KDR and NRP2, which both act on endothelial motility, sprouting, and survival $^{43,44}$. A corresponding TFEA of the DEGs between cEC and sECs showed enrichment of ELK3, KLF6, and SNAI2 (Fig. S3d). The latter is a TF well described in the context of the epithelial-mesenchymal transition ${ }^{45}$, which supports the migrating character of the sEC state. Additionally, ERK and KLF6 control cell proliferation, which was indeed reinstated in ECs after Matrigel transfer ${ }^{46}$. This is further supported by the downregulation of Notch signaling genes in the sprouting cell state, including NOTCH1/4, DLL4, and JAG1 (Fig. S3c). Notch inhibition in cellular model systems has been shown to induce sprouting, branching, and filopodia induction $^{47-49}$. Furthermore, the mTOR pathway proteins, particularly those of the mTOR complex 2 (RICTOR), were strongly downregulated in sECs (Fig. S3c). One downstream target of mTORC2 is the serum and glucocorticoid kinase 1 (SGK1), which is the top upregulated gene in sECs, indicating strong metabolic regulation in this motile cell state ${ }^{50,51}$. 
a

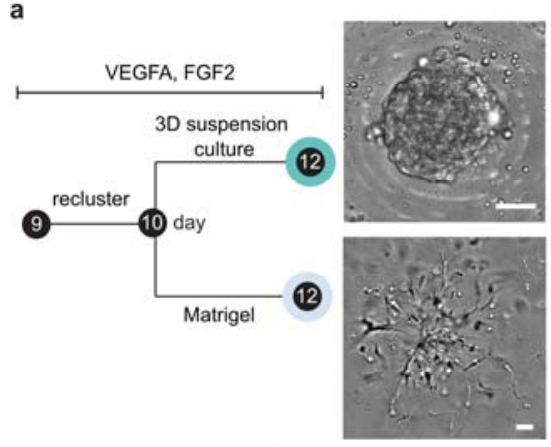

c

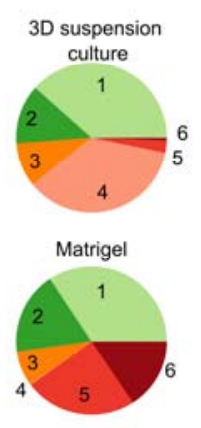

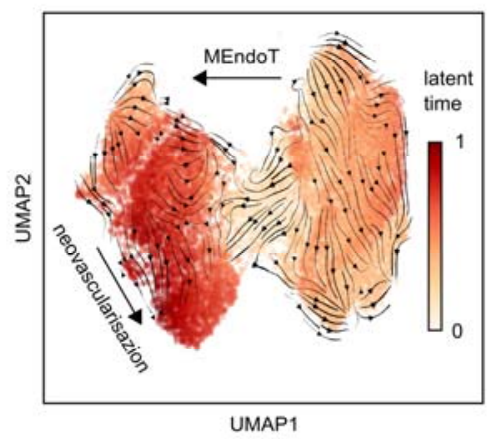

b

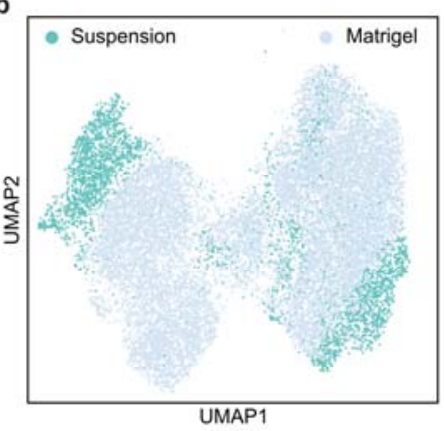

e

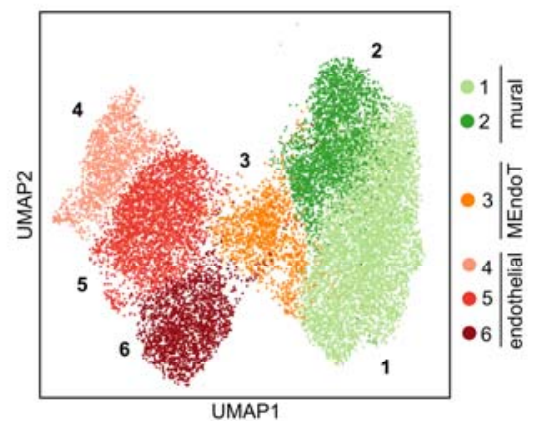

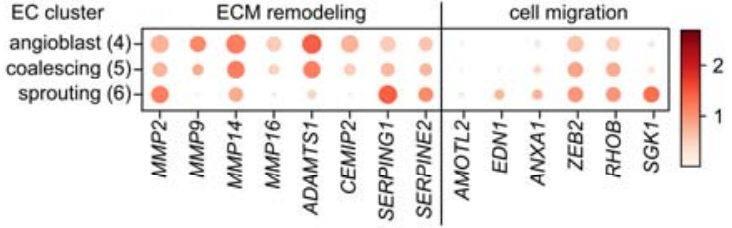

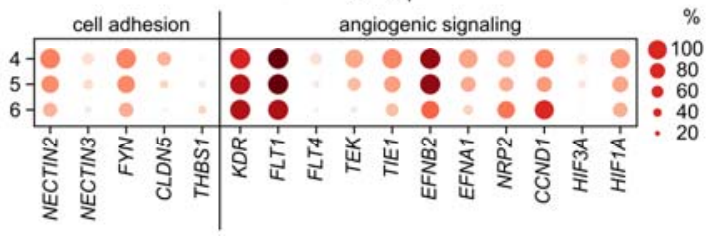

Fig. 4 | Single-cell analysis of hiPSC-derived ECs undergoing neovascularization in Matrigel. a; Experimental overview of the culturing conditions for microvessel formation induction and representative bright-field images of cell culture morphologies. Scale: $50 \mu \mathrm{m}$. b, UMAP plots containing single-cell transcriptomes of cells from 3D suspension and Matrigel cultures. The color code denotes conditions (left) and Leiden cell clusters (right; 1 and 2: mural cells, 3: MEndoT, 4: angioblasts, 5: EC coalescing, 6: EC sprouting). c, Cell type composition of the two culturing conditions is represented as a pie chart. $\mathbf{d}$, Velocity analysis of the single-cell transcriptomic data from a. e, Expression levels of representative DEGs sorted by function for the three EC transcriptional states. Color intensity and dot size denote the normalized cluster mean expression level and the fraction of cell expression for the corresponding gene, respectively.

\section{EC maturation in Matrigel}

Upon prolonging the culturing time, the vessels grew and branched in the Matrigel microenvironment. To determine the genes activated during vessel maturation, we acquired single-cell transcriptomes of day 18 Matrigel cultures. In addition, we investigated the effect of ascorbic acid (AA) on EC maturation. AA increases the synthesis of the basal laminal protein collagen IV and reduces vessel permeability ${ }^{52,53}$. Bright-field imaging showed that vessel length and branching network were comparable in the presence and absence of AA (Fig. 5a). To extract DEGs responsible for EC maturation, single-cell transcriptomes of day 12 and 18 Matrigel cultures (with and without AA) were clustered together (Fig. 5b). Day 18 ECs in the 
presence and absence of $A A$ clustered together, where a low fraction of sprouting and coalescing cells was still observed on day 18 (Fig. 5c). The addition of AA reduced the fraction of SEC in the culture by an order of magnitude. The transcriptomes of the mural and MEndoT cells from day 18 converged partially with the transcriptomes of day 12 and formed an additional cluster. The cell-type composition within the Matrigel culture changed from days 12 to 18 . While the ratio of ECs to mural cells was balanced on day 12, on day 18, the amount of mural and MEndoT cells increased up to $66 \%$. In the presence of $A A$, the fraction of mural cells and MEndoT cells further increased to $85 \%$ (Fig. 5c) due to the increased number of proliferating mural cells (Fig. S5c). The velocity analyses again indicated that the MEndoT population developed from the mural cells towards ECs (Fig. 5d), where day 18 MEndoT cells were further committed to ECs, which is shown by the loss of PDGFRB and further mesenchymal markers (Fig. S4a). Evaluation of the DEGs between ECs revealed that on day 18, ECs increased the expression levels of cell-cell contact genes as ICAMs and CLDN5 (Fig. 5e). Furthermore, the expression of key tubulogenesis genes, such as RASIP1, RHOB, ELMO1 ${ }^{54}$, and ARHGAP29 ${ }^{55}$, were upregulated. In addition, DOCK9 gene expression level increased, which is an RAC1 activator responsible for vascular lateral branching ${ }^{56}$. Based on the expression pattern, we assigned day 18 ECs to tubulogenic EC (tEC). In line with the velocity analysis, a Pearson correlation of the EC transcriptional states from days 12 and 18 showed that the tubulogenic state was closer to the coalescing than to the sprouting state (Fig. S4c). In addition to the changes in the levels of genes controlling the structural change, Notch signaling was upregulated again compared to the sprouting and coalescent states in the forms of NOTCH1, 4 and DLL4. tECs within the +AA sample exhibited a higher latent time within the velocity analysis, indicating a more mature state than without $A A$. Upon increasing the Leiden cluster parameters, it is possible to separate ECs cultured in the presence and absence of $A A$; however, DEG change was minimal. Transcription factor analysis of the DEGs from tECs did not reveal differences in coalescing ECs. 

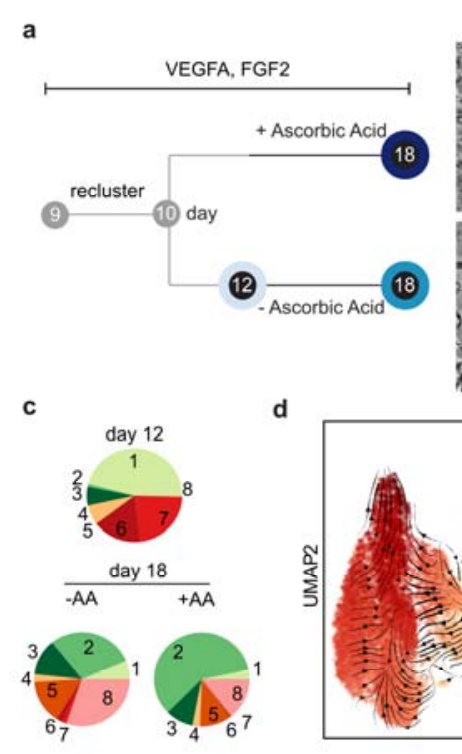

d

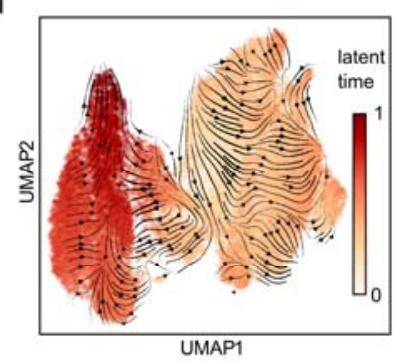

atent
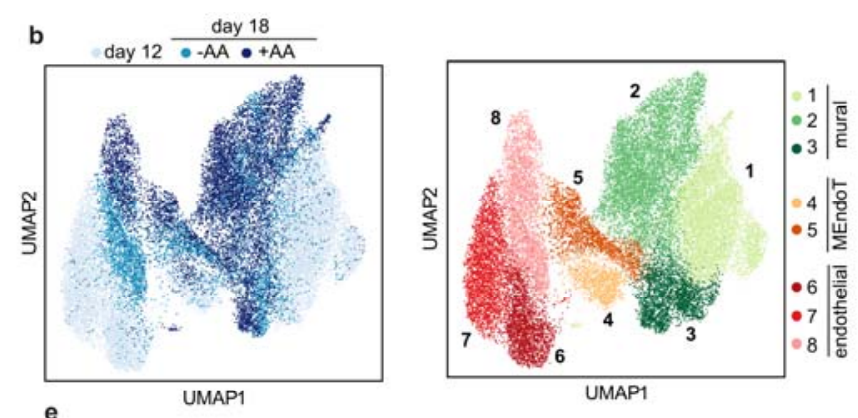

e
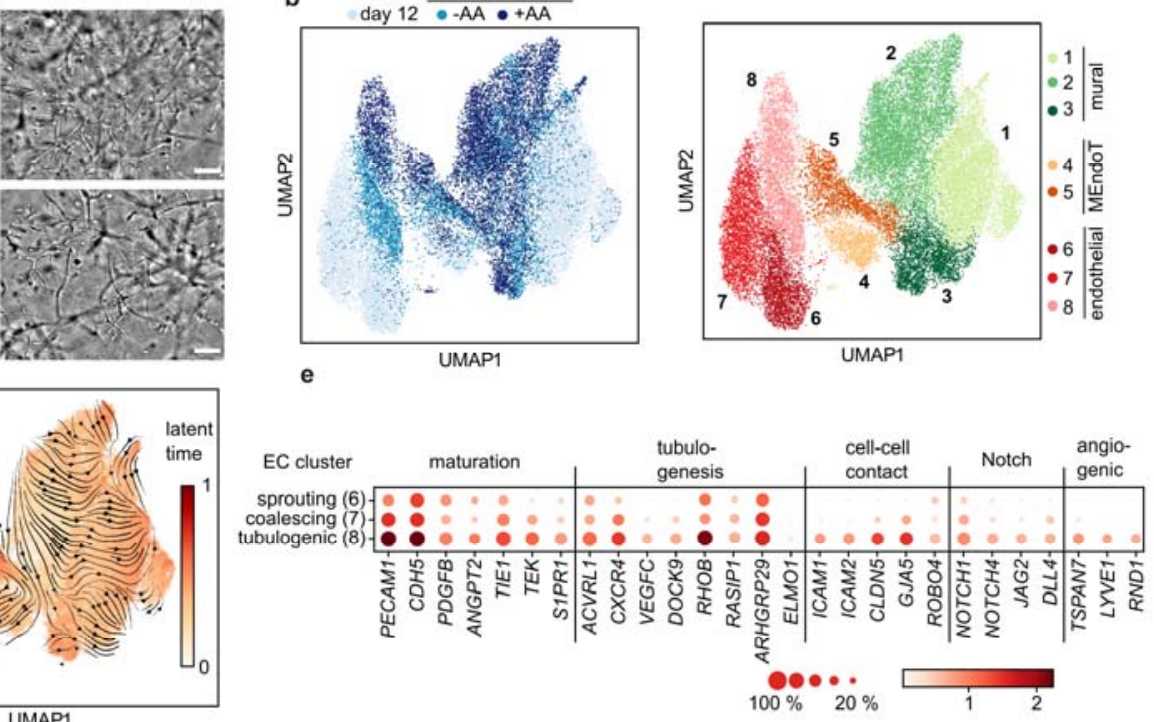

Fig. 5 | Maturation of endothelial cells in Matrigel in presence and absence of ascorbic acid. a, Experimental timeline and applied conditions with corresponding bright-field images of stem cell-derived EC and mural cell cultures in Matrigel on day 18. Scale: $50 \mu \mathrm{m}$. b, Left: UMAP projection of scRNA-seq data from day 12 and two samples from day 18. Right: UMAP projection colored for the annotated cluster 1: mural cell, 2 and 3: pericytes (P1 and P2), 4 and 5: MEndoT, 6: sprouting ECs, 7: coalescing ECs, 8: tubulogenic ECs. The dataset contains 13159, 6373, and 8684 cells for days 12 and 18 without and with ascorbic acid in the media, respectively. c, Cell type composition in the samples. d, Velocity analysis of the single-cell transcriptomes, where the latent time is colored on the UMAP plot. e, Expression levels of representative DEGs sorted by function for the three EC transcriptional states. Color intensity and dot size denote the normalized cluster mean expression level and fraction of cell expression for the corresponding gene, respectively.

\section{Mural and Endothelial cell-cell interaction}

The single-cell transcriptomes of the microvascular culture on day 18 revealed that mural cells of clusters 2 and 3 (Fig. 5b) expressed pericyte markers NG2, RGS5, or NT5E. Among the DEGs were further genes previously found to be enriched in pericytes, such as POSTN and PDLIM3. Therefore, we assigned mural clusters 2 and 3 as pericytes, $\mathrm{P} 1$ and $\mathrm{P} 2$, respectively. While cells of the $\mathrm{P} 1$ cluster expressed the transcription factor FOXF1, cells of the P2 expressed GATA4 (Fig. 6a), where both found in primary pericytes ${ }^{57}$. Localization of the subcellular pericytes within the Matrigel culture failed either due to the low amount of specific antibodies for the DEGs and cross expression of the marker. Mural cells of cluster 1, which consisted mainly of cells from day 12 and clustered with mural cells of the $3 D$ suspension culture. The higher expression level for the mesenchymal TF HAND1/2 and ACTA2, arguing 
that mural cells of cluster 1 were in a premature stage. The velocity analysis of the mural cell transcriptomes, however did not show any direction or evolving latent time underlining the plasticity of the cells.

In the last step, we asked whether it is possible to infer cell-cell communication signals leading to vessel maturation from the scRNA datasets of high plastic cell types, stability, reduced proliferation, and migration ${ }^{58,59}$. To trace cell-cell signaling between pericytes and $\mathrm{tECs}$, we performed a ligand-receptor analysis on the single-cell transcriptomic data. Algorithms evaluating only ligand-receptor expression profiles could not recapitulate literature confirmed signals between ECs and pericytes, for example, TGFB1 or VWF (Fig. S6a and b) in our dataset. Therefore, we performed a NicheNet analysis ${ }^{60}$, which infers ligand-target links between interacting cells by combining DEG data with prior knowledge of signaling and gene regulatory networks (Fig. 6b). NicheNet was applied bidirectionally by investigating ECs as ligand sender and receiver cells. According to the NicheNet results, that is, ligand activities and receptor and target gene interaction potential, were further filtered for gene expression levels to increase the probability of finding relevant interaction links (Fig. 6c). For ECs as senders and pericytes as receivers, the algorithm predicted strong ligand activity for TGFB1, BMP4, and $V W F$. All three have been reported previously to be central for communication between both cell types $^{61}$. TGFB1 acts in paracrine and autocrine signaling, while tECs express the TGFB1 receptor ALK1 (ACVRL1) and pericytes express ALK5 (TGFBR1), which is consistent with current reports using in vitro culture systems ${ }^{62}$. The inferred ligand activity for PDGFB was low and thus filtered out, although PDGFB was highly expressed. This is explained by the fact that we used DEGs of the entire differentiation trajectory to rank ligands, and since PDGFB is expressed strongly at all times, the corresponding target genes within the DEG cannot be expected. In turn, the top predicted ligand activities represented signals that occurred during vessel maturation rather than during vessel formation and the pericyte recruitment phase. Individual NicheNet analysis between ECs and P1 or P2 cells did not differ from the combined analysis. In contrast, the NicheNet analysis for P1 or P2 as signal senders and tECs as the receiver showed that $\mathrm{P} 1$ and $\mathrm{P} 2$ pericytes exhibited shared, as well as individual ligands (Fig. 6d). The shared ligands included CXCL12, ANGPT1, fibronectin, and GDF6, which antagonize VEGF signaling to promote junctional stability and vascular integrity ${ }^{63,64}$. The strongest individual predicted ligand activity of P1 cells was VEGFA, followed by a set of cytokines (IL $1 b$ and IL15) and FGF2. For P2 cells, the ligands with the strongest individual activity were IL24 and FGF5. The corresponding tEC receptors are shown in Fig. 6e and in the detailed table in Fig. S6c. All inferred cytokines have proven pro- or anti-angiogenic function in cancer 
bioRxiv preprint doi: https://doi.org/10.1101/2022 02 15.480506; this version posted February 16, 2022. The copyright holder for this preprint

(which was not certified by peer review) is the author/funder, who has granted bioRxiv a license to display the preprint in perpetuity. It is made available under aCC-BY-NC-ND 4.0 International license.

angiogenesis (SI File); however, their function during neovascularization and vessel maturation is unknown. The resolved heterogeneity of cytokine ligands between pericytes argues for functional differences between P1 and P2 during vessel maturation. 
bioRxiv preprint doi: https://doi.org/10.1101/2022.02 15.480506; this version posted February 16, 2022. The copyright holder for this preprint (which was not certified by peer review) is the author/funder, who has granted bioRxiv a license to display the preprint in perpetuity. It is made available under aCC-BY-NC-ND 4.0 International license.

a

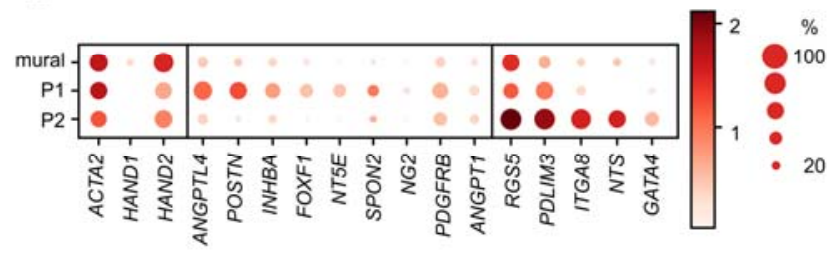

b

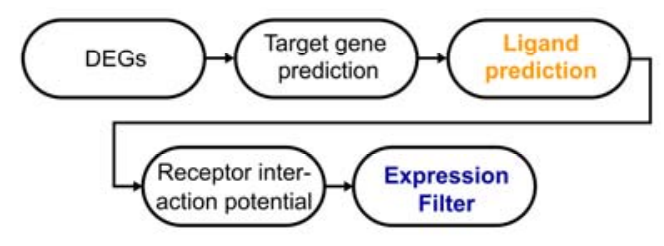

Receptor interaction potential

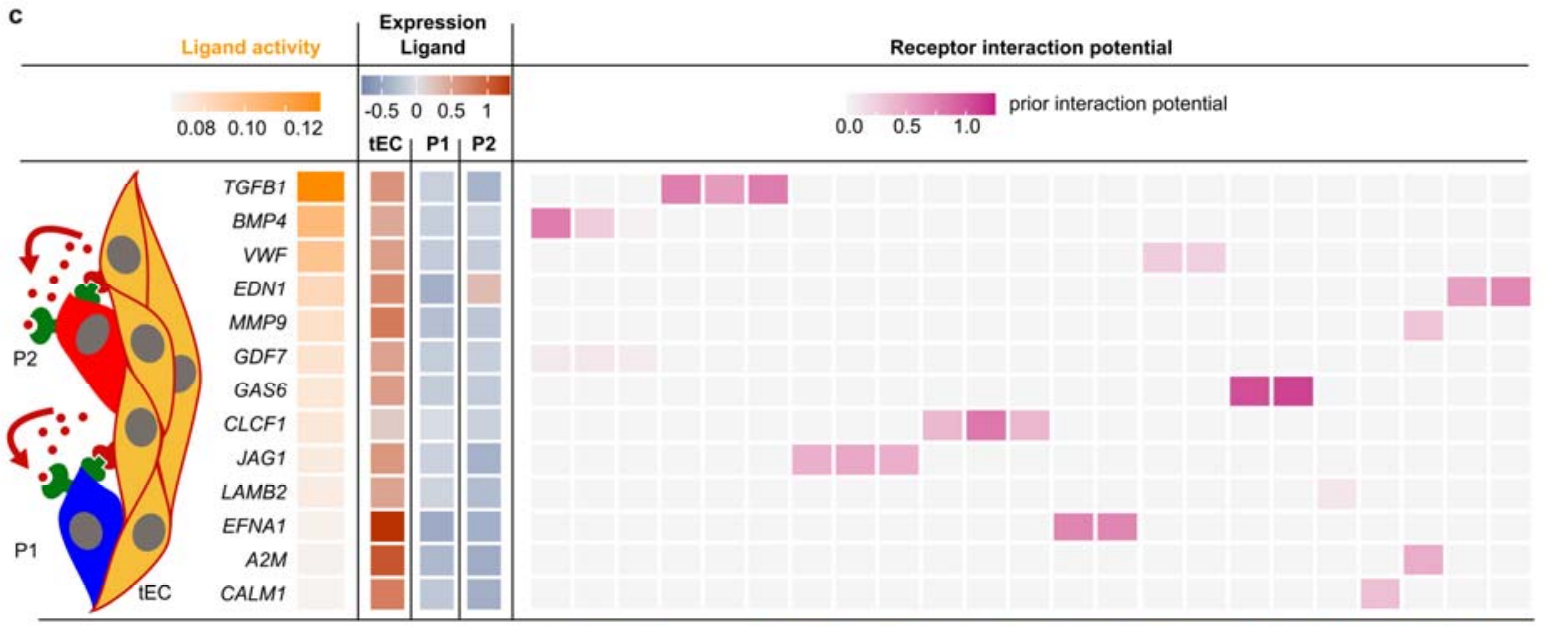

Expression Receptor
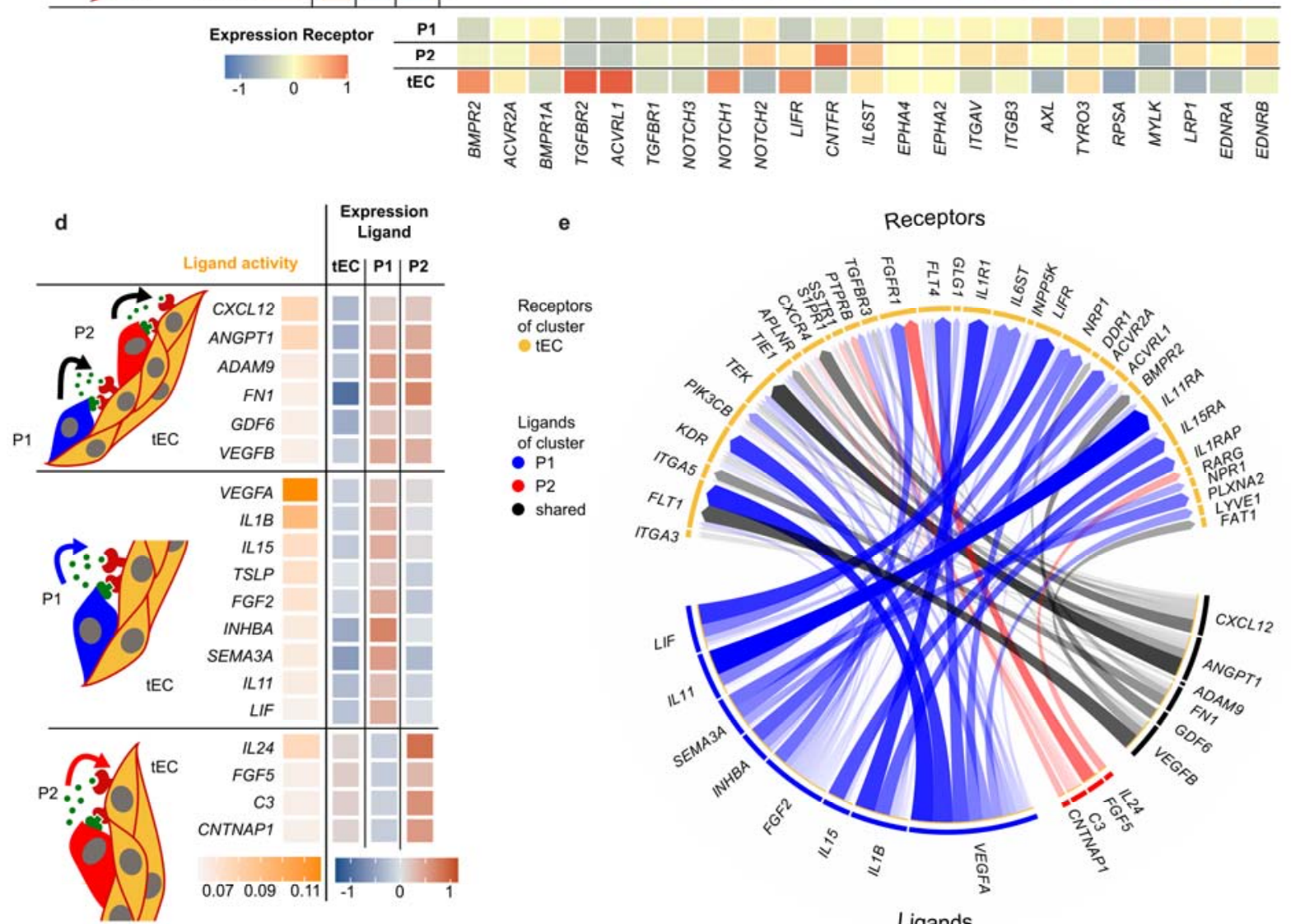

Receptors

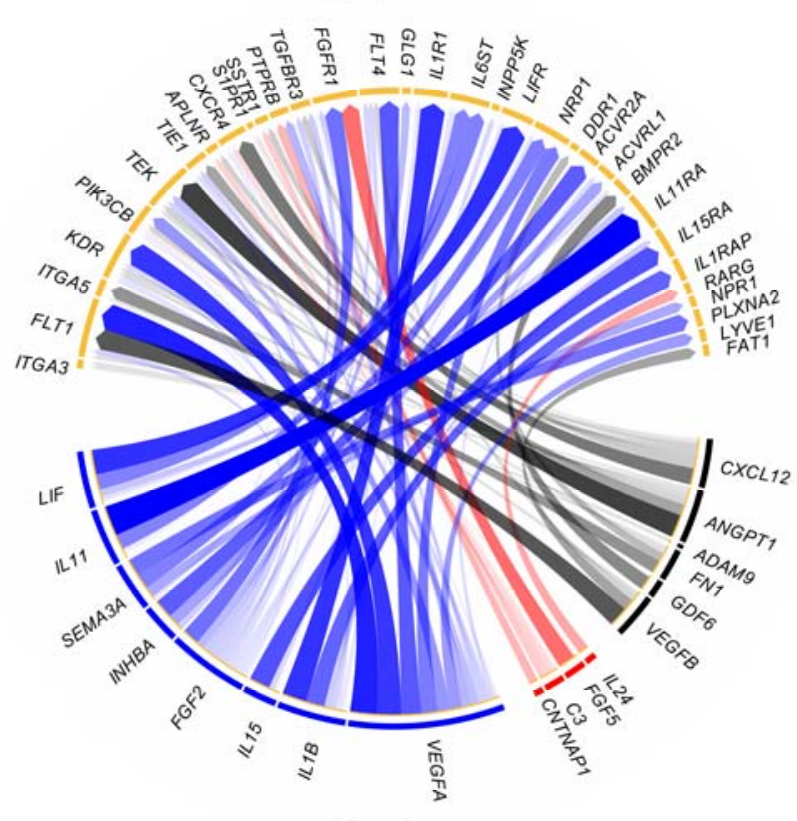

Ligands

Fig. 6 | Inferred signaling between endothelial cells and pericytes during vessel maturation from single-cell transcriptomics. a, Gene expression analysis of the mural cells 
cultured in Matrigel up to day 18 of differentiation. The P1 and P2 clusters show the expression of pericyte markers (bold). Color intensity and dot size denote the normalized cluster mean expression level and fraction of cell expression for the corresponding gene, respectively. b, NicheNet analysis workflow with the expression filter to infer ligand-receptor interactions. c, Ligand-receptor pairs inferred with NicheNet, where single-cell transcriptomes of tEC are used as senders and the combined P1 and P2 pericytes subpopulation as receivers for the analysis. d and e, Ligand-receptor pairs inferred with NicheNet, where single-cell transcriptomes of the P1 and P2 pericyte subpopulations are used as senders and tEC as receiver cells for the analysis. Overlapping (top rows) and unique pericyte ligands (middle and bottom rows) are listed in table format. Ligand-receptor pairs are presented in the circular chord diagram.

\section{Discussion}

Here, we investigated the differentiation of hiPSCs into ECs in a 3D suspension culture, and subsequently, the process of neovascularization with the derived ECs and co-evolving mural cells at the single-cell level. We found that the general differentiation trajectory of ECs and coevolving mural cells in a 3D suspension culture resembled the development of that in a monolayer format. In contrast to the 2D cell culture approach, ECs become quiescent in the 3D suspension culture without entering the neovascularization process. Within the 3D suspension culture the ECM in the surrounding of angioblasts did not contain COL1. Furthermore, COL1 interacting proteins as the ITGA1 receptor were low expressed in angioblasts. ITGA1 signaling is essential for in vivo angiogenesis and could explain the observed proliferation arrest. COL1 expressed by the mural cells could not compensate the missing COL1 since its deposition was spatially restricted to the mural cells in the 3D suspension culture. This also holds true for antiangiogenic factors, such as Lumican (LUM), which colocalized only with mural cells (Fig. S2e). Cell communication between angioblasts and mural cells exists within the 3D suspension culture, which is exemplified by the finding that upon separation from the ECs, the plastic mural cells lost mesenchymal cell type marker expression of PDGFRB. Mesenchymo-angioblast cells express TGFB1 and PDGFB, where the combination of these two factors is used to induce vascular smooth muscle cell development in vitro in the same mesodermal precursor cells ${ }^{16}$. Interestingly, stimulation of mesenchymo-angioblasts with ActivinA (INHBA), a member of the TGFB family, and PDGFB led directly to PDGFRB ${ }^{+}$mural cells as reported before. Additionally to the PDGFRB ${ }^{+}$cells, we detected in the differentiation approach a fraction of $7 \%$ of PECAM ${ }^{+}$ cells on day six and $6 \%$ on day nine (Fig. S7a and b). This demonstrates that EC and mural cells co-evolve in both differentiation directions in vitro. Lineage specification on day 6 of differentiation into an arterial tone could be observed when taking the stronger expression level 
of EFNB2 into account ${ }^{65}$ (Fig. 1C). For scaling EC production with a 3D suspension culture, the transcriptional cell type analysis argues that six days of differentiation is optimal since it shows the largest EC to mural cell ratio.

Transfer of the 3D suspension culture into Matrigel induced sprouting, where the collagen 1 type hydrogel was sufficient but showed a slower growth rate (data omitted). Single-cell transcriptomics untangle coalescence, sprouting, and tubulogenic EC cell states during neovascularization. In comparison to the in vivo reported data, after laser ablation in the choroid layer of mouse eyes, the cellular complexity is far lower. In addition to the expected VEGF and Notch signaling pathways, the mTOR pathway is regulated during neovascularization. mTOR kinase controls various processes, when complexed in the mTORC1, it controls predominantly the cell metabolism. The metabolism as for example a switch between OXPHOS and glycolysis for sECs could not be observed (Fig. S3), as described for tip cells in vivo. However, in sECs, mTORC2 adaptor proteins were downregulated and the downstream effector kinase SGK1 was strongly upregulated, which controls cell survival during angiogenesis ${ }^{66}$ and EC shape ${ }^{67}$; its ablation led to reduced neovascularization and impaired cell migration ${ }^{50}$. The function and regulation of SGK1 through mTORC2 are unknown but have become the focus of further investigations.

Vessel structure formation within the hydrogel culture was accompanied by a change in ECMintegrin expression level. While ITGA2 was upregulated in SEC, a gradual decrease in ITGA1 accompanied by an increase in ITGA1, A5, and $V$ was detected along the cEC to tEC cell stages. Blocking collagen-binding ITGA1 and $A 2$ with antibodies has been shown to reduce angiogenesis ${ }^{68}$. The EC and mural cell stage-assigned integrin profiles provide new dynamic insights. The addition of $\mathrm{AA}$ to the cell culture media increased the expression level and deposition of the central basal lamina protein COL4 (Fig. S5). Therefore, the AA-induced maturation of ECs increased the proliferation rate of mural cells. This may be unwanted in longterm cultures during tissue engineering with no proliferative cells. ECs on day 18 of differentiation did not show a specification marker for arterial or any other vascular cell type. The remaining plasticity of the ECs can be once more exemplified by disseminating of the Matrigel culture and reformation of the 3D suspension culture, where corresponding single-cell transcriptomes show the interconversion of the cell states (Fig. S7e and f).

The most compelling results of the single-cell analysis during the vessel maturation phase in a reduced in vitro microenvironment were the resolved cell-cell communication signals between the two transcriptional pericyte subpopulations and tECs. Pericytes are recruited by PDGFB signaling to capillary walls to stabilize integrity and tube assembly ${ }^{58}$. The directionality of the 
NicheNet ligand-receptor analysis allowed for the resolution of TGFB1 signals in the sender and receiver cells. Next to the known EC-pericyte signaling factors, the complex cytokine profiles of the pericytes were most obvious. The maturation factor for pericyte formation is deciphered as the CXCR4/CXCL12 signaling axis, but in addition to a variety of chemokines with unreported functions during the neovascularization process ${ }^{69}$. Using the DEGs calculated from transcriptional time trajectories within the sender-receiver model, it is possible to extract cell-cell communication signals at defined processes, as applied in the vessel maturation phase.

The present single-cell transcriptomic data and analysis of the vascular structure formation process can be used as a benchmark set for future in vitro vascularization approaches, differentiation attempts to alter the specification of ECs, or investigation into disease-specific gene functions. 


\section{Methods \\ 2D hiPSC cell culture}

HiPSCs were cultured on hESC Matrigel-precoated 6-plates according to manufacturer's recommendations (Corning) in mTeSR1 medium (Stemcell Technologies) at 5\% CO2, 5\% O2, and $37^{\circ} \mathrm{C}$ with daily medium change and split twice a week in a $1: 6$ ratio using $0.05 \%$ TrypsinEDTA (Sigma).

\section{D suspension culture}

For the transfer into a 3D hiPSC cell culture, the medium was aspirated, cells were washed with $2 \mathrm{~mL}$ PBS -/- and $500 \mu \mathrm{L}$ Accutase were added. During incubation at $37^{\circ} \mathrm{C}$ for 3 to $7 \mathrm{~min}$, cells detached while adding $2.5 \mathrm{~mL}$ mTeSR stopped the reaction. The well was washed with $1 \mathrm{~mL}$ mTeSR before centrifugation for $5 \mathrm{~min}$ at $200 \mathrm{xg}$ and resuspended in $500 \mu \mathrm{L}$ meSR with Rock inhibitor (1:1000) and 1\% Penicillin/Streptomycin (P/S, Thermo). The cells were incubated in ultra-low attachment plates at $37^{\circ} \mathrm{C}$ and $100 \mathrm{rpm}$ (Fisher).

\section{$3 D$ suspension culture differentiation to ECs}

At day -1 of the differentiation, cells were transferred into low attachment 6 well plates in order to form embryonic bodies $\left(1.5 \times 10^{6}\right.$ cells/well). Differentiation was performed according to ${ }^{11,16}$. Briefly, from day 0 to 3 N2B27 medium supplemented with BMP4 (25 ng/mL) and CHIR $(7.5 \mu \mathrm{M})$ was used without media exchange. Media was changed daily from day 3 to 7 using StemPro-34 with VEGFA (200 ng/mL) and forskolin $(2 \mu \mathrm{M})$. Afterward, StemPro-34 with VEGFA $(30 \mathrm{ng} / \mathrm{mL})$ and FGF2 (30 $\mathrm{ng} / \mathrm{mL})$ was used with exchange after two days.

\section{D microwell chip}

For matrix-free cultivation, the microwell chip platform, described by Wiedenmann et al., was used to allow the growth of 1196 aggregates in paralle ${ }^{70}$. The chip was coated with $10 \%$ Pluronic F-127 (Sigma) overnight and seeded with approximately $1 \times 10^{6}$ cells. StemPro-34 with VEGFA $(100 \mathrm{ng} / \mathrm{mL})$, FGF2 (100 $\mathrm{ng} / \mathrm{mL})$, and FBS (15\%) was used for cultivation. The medium was exchanged every second day.

\section{Hydrogel cell culture}

For cultivation in 24-well plates, $100 \mu \mathrm{L}$ Matrigel were added into each well of the plate that whole ground is covered and incubated at $37^{\circ} \mathrm{C}$ for $1 \mathrm{~h}$. Aggregates were centrifuged at $800 \mathrm{rpm}$ for $5 \mathrm{~min}$ at $4^{\circ} \mathrm{C}$, resuspended in $80 \mu \mathrm{L}$ of Matrigel and spread on top of the first layer. Gel polymerization was done at $37^{\circ} \mathrm{C}$ inside a container with a bit of ice to allow a slow temperature adjustment. After one hour $0.5 \mathrm{~mL}$ media were added. Media composition complies with microwell chip incubation. +AA samples contained $60 \mu \mathrm{g} / \mathrm{mL}$ ascorbic acid. For imaging, the aggregates were embedded in ibidi slides with a number of 10 to 20 per well. The amount of Matrigel and media was adjusted to the smaller volume.

\section{Fluorescence-activated cell sorting (FACS)}

Harvested cells were washed three times with PBS (200 x g for $5 \mathrm{~min}$ ) and singularized using accutase. Five volumes of FACS buffer (10\% FBS in PBS) were added and centrifuged at $300 \mathrm{xg}$ for $5 \mathrm{~min}$. Blocking was done for $20 \mathrm{~min}$ with StemPro34 + $10 \%$ FBS on ice. The cell suspension was filtered using a $70 \mu \mathrm{m}$ nylon cell strainer (Corning). Live dead staining was performed with Trypan Blue while cells were counted. $20 \mu \mathrm{L}$ of each antibody (FITC Mouse AntiHuman CD31 (BD Pharmingen ${ }^{\mathrm{TM}}$, 555445) and PE Mouse Anti-Human CD140b (BD 
Pharmingen $\left.{ }^{\mathrm{TM}}, 558821\right)$ ) and $1 \mu \mathrm{L}$ of violet fluorescent reactive dye (Invitrogen, REF: L34963A, LOT: 2179253 ) were added per $10^{6}$ cells and incubated for $30 \mathrm{~min}$ at room temperature (RT). After washing once with FACS buffer, cells were resuspended in Tyto buffer $\left(10^{6} \mathrm{cells} / \mathrm{mL}\right.$, MACSQuant Tyto (TM) Running Buffer - Milteniy (Cat. No: 130-107-207; Lot: 5200608355)). The sorting was done on a MACSQuant ${ }^{\circledR}$ Tyto $^{\text {TM }}$. For ensuring unspecific binding, FITC Mouse IgG1, k Isotype Control (BD Pharmingen ${ }^{\mathrm{TM}}$, 554679) and PE Mouse IgG2a, $\mathrm{k}$ Isotype Control (BD Pharmingen ${ }^{\mathrm{TM}}$, 559319) were used.

\section{Flow cytometry}

Singularized cells were washed once with PBS (centrifugation conditions: $300 \times \mathrm{g}, 5 \mathrm{~min}$ ). Cells were fixated at RT for $15 \mathrm{~min}$ with $4 \%$ PFA in PBS and afterward washed twice with FACS buffer. $10^{5}$ cells were transferred into a U-bottom-shaped 96-well plate (Greiner) and an antibody $\left(2 \mu \mathrm{L}\right.$ per $15^{5}$ cells) diluted in $100 \mu \mathrm{L}$ FACS buffer was applied per well. After incubation of $30 \mathrm{~min}$ at RT, cells were washed twice with FACS buffer. For the measurement, the pellet was resuspended in $200 \mu \mathrm{L}$ of FACS buffer and transferred through a cell strainer into FACS-tubes ( $5 \mathrm{~mL}$ round-bottom tubes, Coring). Flow cytometry analysis was performed on a MACSQuant@ VYB.

\section{Cell type stability experiments}

On day 6 (Fig. S7c and d), aggregates were harvested and prepared for FACS, ECs $\left(\right.$ PECAM1 $^{+}$, PDGFRB $^{-}$) and VSMCs (PDGFRB ${ }^{+}$, PECAM1 $^{-}$) were sorted. $2 \times 10^{5}$ of the sorted cell types and the unsorted mixture were seeded and cultured in 6-well plates coated with fibronectin bovine (LIFE Technologies). Medium composition was equivalent to the 3D differentiation.

\section{Cryoembedding}

For cryoembedding of 3D suspension culture aggregates, $500 \mu \mathrm{L}$ of $4 \%$ PFA were added and incubated for $15 \mathrm{~min}$ on ice. The disc was detached from the walls with a needle. After two washing steps with PBS, an incubation with, first, $10 \%$, second, $30 \%$ sucrose at RT for $2 \mathrm{~h}$ and, third, with a $1: 1$ mixture of $30 \%$ sucrose and OCT medium at $4^{\circ} \mathrm{C}$ overnight followed. All incubation steps were implemented on a wave shaker at $47 \mathrm{rmp}$. The medium was replaced by pure OCT and the sample frozen on dry ice. The Slicing was done with a Leica CM1860 cryostat and a thickness of $20 \mu \mathrm{m}$.

\section{Fluorescence imaging}

Slides were washed with PBS, permeabilized in PBS with $0.1 \%$ Triton for 30 min at RT, washed with $0.2 \%$ Tween 20 in PBS (PBST) and blocked with 2\% BSA (Proliant) in PBST for $1 \mathrm{~h}$. Antibodies were applied in the concentration as recommended by the manufacturer (specifications in SI File) in the blocking solution. After primary and secondary antibody staining, five washing steps with 5 min PBST were performed. Prior to confocal imaging (Zeiss Axio Observer LSM 880), Vectashield® Mounting Medium was added to the sample, it was covered and sealed by a coverslip.

Image analysis

IF and bright-field images were corrected for brightness and contrast with ImageJ. Z-projection of fluorescence images was performed using maximal intensity. ImageJ version 1.52p was used $^{71}$. 


\section{Sample preparation for scRNA-seq}

Matrix-free samples were washed with PBS, resuspended in Accutase and incubated for cell detachment at $37^{\circ} \mathrm{C}$ for $30 \mathrm{~min}$. The reaction was stopped by adding five volumes of media. Afterward, cells were washed once with PBS. Prior to this process, Matrigel embedded samples were washed with PBS, incubated with $0.5 \mathrm{~mL}$ Collagenase/Dispase Solution (1:100 to a final concentration of $1 \mathrm{mg} / \mathrm{mL}$ in StemPro34) for around $4 \mathrm{~h}$, until organoids detached from the Matrigel. The enzymatic reaction was stopped with $1 \mathrm{~mL}$ Neutralisation Buffer $(1 \% \mathrm{BSA}, 1 \% \mathrm{P} / \mathrm{S}$ in DMEM:F12). The single cells were cryo-preserved in DMEM with $10 \%$ heat-inactivated FBS (Thermo Fisher Scientific) and 10\% DMSO based on a previously described scRNA-seq sample

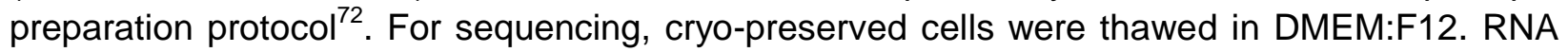
libraries were generated using Chromium Single Cell 3' library and gel bead kit v3.1 (10x Genomics). The amplified cDNA library was sequenced on a NovaSeq 6000 S2 flow cell from Illumina. The sequenced cell numbers can be found in Table S1.

\section{ScRNA-seq data pre-processing}

Sequencing raw files were demultiplexed, aligned (reference genome hg38_ensrel97), filtered, barcodes and UMIs counted, and subjected to a quality filter with CellRanger (version 3.0.1, 10xGenomics). The pre-processing and downstream analysis were performed with the package 'Scanpy API' in python with default parameters, if not stated differently ${ }^{73}$. First, dead or stressed cells, identified by a percentage of mitochondrial genes higher than $10 \%$, were filtered out. Next, cells with less than 200 and genes expressed in less than three cells were excluded. Afterward, the datasets of different days and experiments were concatenated, normalized to $10^{4}$ gene counts per cell and log-transformed. Batch effects were corrected using ComBat. Further on, the top 4,000 highly variable genes were used for the downstream analysis. As discussed by Luecken and Theis, we corrected for the total gene counts, percentage of mitochondrial genes, and the cell cycle distribution of $\mathrm{S}, \mathrm{G} 2$ and $\mathrm{M}$ phase to investigate differentiation-dependent changes on the transcriptome level ${ }^{17}$.

\section{Dimensionality reduction, clustering, and cell-type annotation}

The single-cell nearest neighborhood graph was computed with the first 50 principal components and ten nearest neighbors. The cells were clustered with the Leiden algorithm with a resolution of 0.5 . For visualization, the dimensionality of the data was reduced using Uniform Manifold Approximation and Projection (UMAP) ${ }^{74}$. For cell-type annotation, 300 DEGs for each of the clusters were calculated by ranking the clusters against all remaining cells with the t-test method (SI File). Clusters with proteasome-related genes scored at the top or a significantly reduced gene count were removed from the dataset as representing dying or damaged cells. The remaining clusters were annotated based on known marker genes.

\section{RNA velocity through dynamical modeling}

We analyzed the RNA velocity to investigate developmental trajectories by recovering directed dynamic gene information through splicing kinetics. Information like clustering and UMAP coordinates were retrieved from the Scanpy analysis. The pre-processing and downstream analysis were performed with scVelo using default parameters ${ }^{20}$. Splice variants and cells were filtered, normalized, and logarithmized with the function scv.pp.filter_and_normalize (parameters: min_cells=3, min_counts $=200$, min_shared_counts $=20$, n_top_genes $=5500$ ). The 
moments based on the connectivities were calculated with 30 PCAs and 30 neighbors in the next step. After recovering the dynamics, the latent time was calculated and the velocity was calculated as a dynamical model.

Integration of datasets from different sequencing approaches

For integration and correction of datasets from different sequencing runs (Fig. 3, Fig. S1, Fig. S2, and Fig. S7), we applied bbknn to the datasets (neighbors_within_batch=20, n_pcs=30, trim=0, copy=True). We then reclustered the cells with the Leiden algorithm at a resolution of $0.8^{75,76}$.

\section{Transcription factor enrichment analysis}

The identified cluster-specific DDGs or DEGs were entered in the ChEA3 web tool ${ }^{77}$ and the mean rank was plotted using $R$.

\section{Pathway and Gene Ontology enrichment}

DEGs were filtered by their unique expression over all clusters (standard deviation above 0.5) and an expression value above 0.5. For the GO term enrichment, the $R$ package enrichR was used with the "GO Biological Process 2018" database and plotted in $\mathrm{R}^{78,79}$.

\section{CellphoneDB}

The count matrix and cluster annotation were exported from scanpy, imported into $\mathrm{R}$, and processed as recommended by the authors ${ }^{80}$. Cell-cell interactions were selected by the highest mean score and lowest $p$-value.

\section{NicheNet}

As Target gene input, top 300 DEGs have been used. The calculation was done in $R$, converting the anndata element into a Seurat object. The process was performed as recommended by the authors ${ }^{60}$.

\section{Software specifications}

The scRNA-seq alignment was run in CellRanger version 3.0.1 and the analyses were run in python 3.7.4 with Scanpy API version 1.4.4 or 1.5.1, anndata version 0.6.22 or 0.7.4, umap version 0.3 .10 , numpy version 1.17 .4 , scipy version 1.5 .1 , pandas version 0.25 .3 or 1.0 .5 , scikitlearn version 0.22 , statsmodels version 0.10 .1 , python-igraph version 0.7 .1 , scvelo version 0.2.1, matplotlib version 3.2.1, seaborn version 0.9 .0 , loompy version 3.0.6, XlsxWriter version 1.2.6, bbknn version 1.3.6 and scrublet version 0.2.1.

The plots of TFEA and GO term analysis were generated in RStudio with $\mathrm{R}$ version 3.6 .0 with the usage of the $R$ packages enrichR_3.0, ggpubr_0.4.0, ggplot2_3.3.3, stringr_1.4.0, EBImage_4.32.0, and bioimagetools_1.1.5.

NicheNet analysis was performed using following package versions: xlsx_0.6.5, ggpubr_0.4.0.999, cowplot_1.1.1, RColorBrewer_1.1-2, circlize_0.4.13, forcats_0.5.1, stringr_1.4.0, dplyr_1.0.7, purrr_0.3.4, readr_2.1.1, tidyr_1.1.4, tibble_3.1.0, ggplot2_3.3.5, tidyverse_1.3.1, SeuratObject_4.0.4, Seurat_4.0.2, nichenetr_1.0.0, gridBase_0.4-7, and ComplexHeatmap_2.6.2 


\section{Data availability}

Raw data will be available via Gene Expression Omnibus.

\section{Code availability}

The code for scRNA-seq analysis will be available on github.

\section{References}

1. Ricard, N., Bailly, S., Guignabert, C. \& Simons, M. The quiescent endothelium: signalling pathways regulating organ-specific endothelial normalcy. Nat. Rev. Cardiol. 202118818 , 565-580 (2021).

2. Chatterjee, S. Endothelial signaling in vascular dysfunction and disease $\square$ : from bench to bedside.

3. Dejana, E., Hirschi, K. K. \& Simons, M. The molecular basis of endothelial cell plasticity. Nat. Commun. 201781 8, 1-11 (2017).

4. Holm, A., Heumann, T. \& Augustin, H. G. Microvascular Mural Cell Organotypic Heterogeneity and Functional Plasticity. Trends Cell Biol. 28, 302-316 (2018).

5. Nguyen, J., Lin, Y.-Y. \& Gerecht, S. The next generation of endothelial differentiation: Tissue-specific ECs. Cell Stem Cell 0, (2021).

6. Poole, T. J., Finkelstein, E. B. \& Cox, C. M. The role of FGF and VEGF in angioblast induction and migration during vascular development. Dev. Dyn. 220, 1-17 (2001).

7. Tan, J. Y., Sriram, G., Rufaihah, A. J., Neoh, K. G. \& Cao, T. Efficient derivation of lateral plate and paraxial mesoderm subtypes from human embryonic stem cells through GSKimediated differentiation. Stem Cells Dev. 22, 1893-1906 (2013).

8. Chen, G. et al. Chemically defined conditions for human iPSC derivation and culture. Nat. Methods 8, 424-429 (2011).

9. Xu, M., He, J., Zhang, C., Xu, J. \& Wang, Y. Strategies for derivation of endothelial lineages from human stem cells. Stem Cell Res. Ther. 2019101 10, 1-14 (2019).

10. Paik, D. T. et al. Single-Cell RNA Sequencing Unveils Unique Transcriptomic Signatures of Organ-Specific Endothelial Cells. Circulation 142, 1848-1862 (2020).

11. Olmer, R. et al. Differentiation of Human Pluripotent Stem Cells into Functional Endothelial Cells in Scalable Suspension Culture. Stem Cell Reports 10, 1657-1672 (2018).

12. Wimmer, R. A. et al. Human blood vessel organoids as a model of diabetic vasculopathy. Nature 565, 505-510 (2019).

13. McCracken, I. R. et al. Transcriptional dynamics of pluripotent stem cell-derived endothelial cell differentiation revealed by single-cell RNA sequencing. Eur. Heart J. 41, 1024-1036 (2020).

14. Noden, D. M. Embryonic origins and assembly of blood vessels. Am. Rev. Respir. Dis. 140, 1097-1103 (1989).

15. Rohlenova, K. et al. Single-Cell RNA Sequencing Maps Endothelial Metabolic Plasticity in Pathological Angiogenesis. Cell Metab. 31, 862-877.e14 (2020). 
16. Patsch, C. et al. Generation of vascular endothelial and smooth muscle cells from human pluripotent stem cells. Nat. Cell Biol. 17, 994-1003 (2015).

17. Luecken, M. D. \& Theis, F. J. Current best practices in single-cell RNA-seq analysis: a tutorial. Mol. Syst. Biol. 15, (2019).

18. Vodyanik, M. A. et al. A Mesoderm-Derived Precursor for Mesenchymal Stem and Endothelial Cells. Cell Stem Cell 7, 718-729 (2010).

19. La Manno, G. et al. RNA velocity of single cells. Nature 560, 494-498 (2018).

20. Bergen, V., Lange, M., Peidli, S., Wolf, F. A. \& Theis, F. J. Generalizing RNA velocity to transient cell states through dynamical modeling. Nat. Biotechnol. 20203812 38, 14081414 (2020).

21. Paul, J. D. et al. SLIT3-ROBO4 Activation Promotes Vascular Network Formation in Human Engineered Tissue and Angiogenesis in Vivo. J. Mol. Cell. Cardiol. 64, 124-131 (2013).

22. Reuten, R. et al. Structural decoding of netrin-4 reveals a regulatory function towards mature basement membranes. Nat. Commun. 2016 717, 1-17 (2016).

23. Eichmann, A. \& Simons, M. VEGF signaling inside vascular endothelial cells and beyond. Curr. Opin. Cell Biol. 24, 188-193 (2012).

24. Puri, M. C., Partanen, J., Rossant, J. \& Bernstein, A. Interaction of the TEK and TIE receptor tyrosine kinases during cardiovascular development. Development 126, 45694580 (1999).

25. Ohnuki, H. et al. BAZF, a novel component of cullin3-based E3 ligase complex, mediates VEGFR and Notch cross-signaling in angiogenesis. Blood 119, 2688-2698 (2012).

26. Pham, V. N. et al. Combinatorial function of ETS transcription factors in the developing vasculature. Dev. Biol. 303, 772-783 (2007).

27. Kim, K. S. et al. ELK3 expressed in lymphatic endothelial cells promotes breast cancer progression and metastasis through exosomal miRNAs. Sci. Reports 201991 9, 1-10 (2019).

28. Birdsey, G. M. et al. Transcription factor Erg regulates angiogenesis and endothelial apoptosis through VE-cadherin. Blood 111, 3498-3506 (2008).

29. Osterwalder, M. et al. HAND2 targets define a network of transcriptional regulators that compartmentalize the early limb bud mesenchyme. Dev. Cell 31, 345-357 (2014).

30. Wu, S.-P., Dong, X.-R., Regan, J. N., Su, C. \& Majesky, M. W. Tbx18 Regulates Development of the Epicardium and Coronary Vessels. Dev. Biol. 383, 307 (2013).

31. Martowicz, A. et al. Endothelial $\beta$-Catenin Signaling Supports Postnatal Brain and Retinal Angiogenesis by Promoting Sprouting, Tip Cell Formation, and VEGFR (Vascular Endothelial Growth Factor Receptor) 2 Expression. Arterioscler. Thromb. Vasc. Biol. 39, 2273-2288 (2019).

32. Altschuler, D. L. \& Ribeiro-Neto, F. Mitogenic and oncogenic properties of the small G protein Rap1b. Proc. Natl. Acad. Sci. 95, 7475-7479 (1998).

33. Griffin, J. N. et al. RAPGEF5 regulates nuclear translocation of $\beta$-catenin. Dev. Cell 44, 
248 (2018).

34. Gingras, A. R., Puzon-McLaughlin, W., Bobkov, A. A. \& Ginsberg, M. H. Structural Basis of Dimeric Rasip1 RA Domain Recognition of the Ras Subfamily of GTP-Binding Proteins. Structure 24, 2152-2162 (2016).

35. Fang, J. S. et al. Shear-induced Notch-Cx37-p27 axis arrests endothelial cell cycle to enable arterial specification. Nat. Commun. 201781 8, 1-14 (2017).

36. Wang, Y. et al. Angiomotin-like2 Gene (amotl2) Is Required for Migration and Proliferation of Endothelial Cells during Angiogenesis. J. Biol. Chem. 286, 41095 (2011).

37. Pin, A. L. et al. Annexin-1-mediated Endothelial Cell Migration and Angiogenesis Are Regulated by Vascular Endothelial Growth Factor (VEGF)-induced Inhibition of miR-196a Expression. J. Biol. Chem. 287, 30541 (2012).

38. He, Y. et al. The Cdc42/Rac1 regulator CdGAP is a novel E-cadherin transcriptional corepressor with Zeb2 in breast cancer. Oncogene 20173624 36, 3490-3503 (2017).

39. Herbst, T. J., McCarthy, J. B., Tsilibary, E. C. \& Furcht, L. T. Differential effects of laminin, intact type IV collagen, and specific domains of type IV collagen on endothelial cell adhesion and migration. J. Cell Biol. 106, 1365 (1988).

40. Bhutto, I. A. et al. Localization of Collagen XVIII and the Endostatin Portion of Collagen XVIII in Aged Human Control Eyes and Eyes with Age-Related Macular Degeneration. Invest. Ophthalmol. Vis. Sci. 45, 1544-1552 (2004).

41. Seomun, Y., Kim, J. T. \& Joo, C. K. MMP-14 mediated MMP-9 expression is involved in TGF-beta1-induced keratinocyte migration. J. Cell. Biochem. 104, 934-941 (2008).

42. Sacharidou, A. et al. Endothelial lumen signaling complexes control 3D matrix-specific tubulogenesis through interdependent Cdc42- and MT1-MMP-mediated events. Blood 115, 5259 (2010).

43. Favier, B. et al. Neuropilin-2 interacts with VEGFR-2 and VEGFR-3 and promotes human endothelial cell survival and migration. Blood 108, 1243-1250 (2006).

44. Gerhardt, H. et al. VEGF guides angiogenic sprouting utilizing endothelial tip cell filopodia. J. Cell Biol. 161, 1163 (2003).

45. Cano, A. et al. The transcription factor Snail controls epithelial-mesenchymal transitions by repressing E-cadherin expression. Nat. Cell Biol. 200022 2, 76-83 (2000).

46. Das, A. et al. Disruption of an SP2/KLF6 repression complex by SHP is required for farnesoid X receptor-induced endothelial cell migration. J. Biol. Chem. 281, 39105-39113 (2006).

47. Hellström, M. et al. Dll4 signalling through Notch1 regulates formation of tip cells during angiogenesis. Nat. 20064457129 445, 776-780 (2007).

48. Lobov, I. B. et al. Delta-like ligand 4 (DII4) is induced by VEGF as a negative regulator of angiogenic sprouting. Proc. Natl. Acad. Sci. U. S. A. 104, 3219-3224 (2007).

49. Suchting, S. et al. The Notch ligand Delta-like 4 negatively regulates endothelial tip cell formation and vessel branching. Proc. Natl. Acad. Sci. U. S. A. 104, 3225 (2007).

50. Zarrinpashneh, E. et al. Ablation of SGK1 Impairs Endothelial Cell Migration and Tube 
Formation Leading to Decreased Neo-Angiogenesis Following Myocardial Infarction. PLoS One 8, e80268 (2013).

51. Hong, F. et al. mTOR-Raptor Binds and Activates SGK1 to Regulate p27 Phosphorylation. Mol. Cell 30, 701-711 (2008).

52. Utoguchi, N. et al. Ascorbic acid stimulates barrier function of cultured endothelial cell monolayer. J. Cell. Physiol. 163, 393-399 (1995).

53. Yoshikawa, K., Takahashi, S., Imamura, Y., Sado, Y. \& Hayashi, T. Secretion of nonhelical collagenous polypeptides of alpha1(IV) and alpha2(IV) chains upon depletion of ascorbate by cultured human cells. J. Biochem. 129, 929-936 (2001).

54. Epting, D. et al. The Rac1 regulator ELMO1 controls vascular morphogenesis in zebrafish. Circ. Res. 107, 45-55 (2010).

55. Xu, K. \& Cleaver, O. Tubulogenesis during blood vessel formation. Semin. Cell Dev. Biol. 22, 993 (2011).

56. Abraham, S. et al. A Rac/Cdc42 exchange factor complex promotes formation of lateral filopodia and blood vessel lumen morphogenesis. Nat. Commun. 6, 1-14 (2015).

57. Muhl, L. et al. Single-cell analysis uncovers fibroblast heterogeneity and criteria for fibroblast and mural cell identification and discrimination. Nat. Commun. $202011111,1-$ 18 (2020).

58. Lindahl, P., Johansson, B. R., Levéen, P. \& Betsholtz, C. Pericyte loss and microaneurysm formation in PDGF-B-deficient mice. Science (80-. ). 277, 242-245 (1997).

59. Stratman, A. N., Schwindt, A. E., Malotte, K. M. \& Davis, G. E. Endothelial-derived PDGF-BB and HB-EGF coordinately regulate pericyte recruitment during vasculogenic tube assembly and stabilization. Blood 116, 4720-4730 (2010).

60. Browaeys, R., Saelens, W. \& Saeys, Y. NicheNet: modeling intercellular communication by linking ligands to target genes. Nat. Methods 17, 159-162 (2020).

61. Sweeney, M. \& Foldes, G. It Takes Two: Endothelial-Perivascular Cell Cross-Talk in Vascular Development and Disease. Front. Cardiovasc. Med. 5, 154 (2018).

62. Dave, J. M., Mirabella, T., Weatherbee, S. D. \& Greif, D. M. Pericyte ALK5/TIMP3 Axis Contributes to Endothelial Morphogenesis in the Developing Brain. Dev. Cell 44, 665678.e6 (2018).

63. Krispin, S. et al. Growth Differentiation Factor 6 Promotes Vascular Stability by Restraining Vascular Endothelial Growth Factor Signaling. Arterioscler. Thromb. Vasc. Biol. 38, 353-362 (2018).

64. SALCEDO, R. \& OPPENHEIM, J. J. Role of Chemokines in Angiogenesis: CXCL12/SDF1 and CXCR4 Interaction, a Key Regulator of Endothelial Cell Responses. Microcirculation 10, 359-370 (2010).

65. Zhang, J. et al. Functional characterization of human pluripotent stem cell-derived arterial endothelial cells. Proc. Natl. Acad. Sci. U. S. A. 114, E6072-E6078 (2017).

66. Catela, C., Kratsios, P., Hede, M., Lang, F. \& Rosenthal, N. Serum and glucocorticoidinducible kinase 1 (SGK1) is necessary for vascular remodeling during angiogenesis. 
Dev. Dyn. 239, 2149-2160 (2010).

67. Tsuji-Tamura, K. \& Ogawa, M. Inhibition of the PI3K-Akt and mTORC1 signaling pathways promotes the elongation of vascular endothelial cells. J. Cell Sci. 129, 11651178 (2016).

68. Senger, D. R. et al. The alpha(1)beta(1) and alpha(2)beta(1) integrins provide critical support for vascular endothelial growth factor signaling, endothelial cell migration, and tumor angiogenesis. Am. J. Pathol. 160, 195-204 (2002).

69. Salcedo, R. et al. Vascular endothelial growth factor and basic fibroblast growth factor induce expression of CXCR4 on human endothelial cells. In vivo neovascularization induced by stromal-derived factor-1a. Am. J. Pathol. 154, 1125-1135 (1999).

70. Wiedenmann, S. et al. Single-cell-resolved differentiation of human induced pluripotent stem cells into pancreatic duct-like organoids on a microwell chip. Nat. Biomed. Eng. 5, 897-913 (2021).

71. Schindelin, J. et al. Fiji: an open-source platform for biological-image analysis. Nat. Methods 2012 979, 676-682 (2012).

72. Guillaumet-Adkins, A. et al. Single-cell transcriptome conservation in cryopreserved cells and tissues. Genome Biol. 18, 45 (2017).

73. Wolf, F. A., Angerer, P. \& Theis, F. J. SCANPY: Large-scale single-cell gene expression data analysis. Genome Biol. 19, 15 (2018).

74. Mcinnes, L., Healy, J. \& Melville, J. UMAP: Uniform Manifold Approximation and Projection for Dimension Reduction. (2018).

75. Polański, K. et al. BBKNN: fast batch alignment of single cell transcriptomes. Bioinformatics 36, 964-965 (2020).

76. Traag, V., Waltman, L. \& van Eck, N. J. From Louvain to Leiden: guaranteeing wellconnected communities. Sci. Rep. 9, (2018).

77. Keenan, A. B. et al. ChEA3: transcription factor enrichment analysis by orthogonal omics integration. Nucleic Acids Res. 47, W212-W224 (2019).

78. Kuleshov, M. V. et al. Enrichr: a comprehensive gene set enrichment analysis web server 2016 update. Nucleic Acids Res. 44, W90-W97 (2016).

79. Chen, E. Y. et al. Enrichr: Interactive and collaborative HTML5 gene list enrichment analysis tool. BMC Bioinformatics 14, 128 (2013).

80. Efremova, M., Vento-Tormo, M., Teichmann, S. A. \& Vento-Tormo, R. CellPhoneDB: inferring cell-cell communication from combined expression of multi-subunit ligandreceptor complexes. Nat. Protoc. 15, 1484-1506 (2020).

\section{Acknowledgements}


This work is supported by the Helmholtz Pioneer Campus, and ERC (Consolidator Grant Number 772646), We thank Thomas Walzthöni for bioinformatics support provided at the Bioinformatics Core Facility, Institute of Computational Biology, Helmholtz Zentrum München.

\section{Author contributions}

S.R., M.A., and M.Meier. designed the study. S.R., M.A., C.B., M.Marder., and M.Meier. executed the biological experiments. S.R., M.A. did the imaging and image analysis. S.R. S.W. performed the scRNA-seq analysis. S.U., F.T. and M.Meier supervised the study. The manuscript was written by S.R., and M.Meier. All authors corrected and approved the paper.

\section{Competing interests}

The authors declare no competing interests. 